for establishment of a new population, and that migration might be of less importance than changes in situ.

Theories of evolution were divided by Prof. R. A. Fisher into two kinds: those that explain adaptations, and those that fail to account for them. The development of the living organism must account for adaptations, otherwise (1) species must arise by an inner urge causing a progressive predetermined course undeterred by differences of birth or death, (2) the environment must govern the course of change. The "mutation theory, once popular among geneticists", omits the consideration of adaptations, and fails to explain the functions of organisms or their parts.

Dr. R. N. Salaman, in a few words at the end of the discussion, showed that a mutation might arise which only in certain cireumstances might be of direct value to a species. A Solanum from Mexico, a country free from wart disease, has in Great Britain de. veloped a recessive, bred true, which is immune to wart: a character that is useless in its native country but of the utmost value for England.

The value of protective adaptations in mimicry among arthropods was stressed by Prof. G. D. Hale Carpenter. Mimetic effects are produced in a variety of ways all serving the same end; a characteristie product of natural selection. Polymorphism in the case of mimicry is favoured by natural selection, contrary to the views of Dr. Turrill. A species gains by resemblance to different models or many kinds of inanimate objects, as this increases the work the enemy has to do in finding food. Habit plays an important part; conspicuovs species furnished with unpleasant qualities have habits displaying them; mimies have habits enhancing the likeness, but often abandoned in real danger. The fact that mimicry deceives the artist but not the anatomist can only be explained by natural selection. The correspondence between models and mimicry in precise distribution is of utmost importance.

The question of numbers was discussed by Prof. J. B. S. Haldane. We are not in a position to detect evolutionary changes in a population at our disposal. Palæontologists agree that at least 20,000 generations are required to produce a recognisable change. If a gene is increased by 0.01 per cent, its frequency will increase in a random population from 0.01 to 99 per cent in 23,400 generations; but it would need a population of four millions to reveal it. This point had been also considered by Prof. Ruggles Gates, who said that in quite small populations the conditions would scarcely differ from ehance survivals.

Prof. Haldane also said that new genes must be such as to decrease fitness, for if not they would already have spread through a population. The primary role of natural selection is to stabilise a species. Evolutionary changes are only to be expected as a result of drastic changes in environment; there is rarely direct evidence of selection. But such evidence exists in man, in the case of resistance to a disease : the progressive diminution of tuberculosis is due to natural selection developing immunity.

Parallel mutation was discussed by Prof. Ruggles Gates : it is so abundant, causing parallel evolution, that we must look on phylogeny not as like the usual Darwinian tree, but as the root system of a fig-tree which sends down parallel roots, descending and interlacing.

The facts of mimicry in insects, however, according to Prof. Hale Carpenter, cannot be explained by parallel mutation. The results in mimics and models are not the same, and similar effects are produced in different ways. One of a series of forms of a polymorphic mimetic species, of which the majority mimic species of one subfamily, may mimic a species of quite a different subfamily, and yet this form is linked with the others by intermediates.

\title{
The Mysterious Number 137
}

$\mathrm{I}^{\mathrm{T}}$ is a remarkable fact, first made prominent by Sommerfeld's discussion of the fine structure of the hydrogen spectrum, that from three physical constants, $h$ (Planck's constant), $c$ (the velocity of light in vacuo) and $e$ (the charge on an electron), a dimensionless pure number can be formed, which usually occurs in the form $h c / 2 \pi e^{2}$, with the numerical value 137 , or more accurately $137 \cdot 2$.

Dr. Max Born, in a lecture delivered to the South Indian Science Association at Bangalore on November 9, 1935 (Proc. Indian Acad. Sci., 2, 533; 1935), declared that the explanation of this number must be the central problem of natural philosophy. Its existence can be ascribed to the fact that there are two different 'natural' units of length, a larger one $\lambda_{0}=h / m c$ (the so-called Compton wave-length) taken from quantum theory, and a smaller one $a_{0}=e^{2} / m c^{2}$ (the so-called radius of the electron). Their ratio is $2 \pi$ times the mysterious number. After pointing out the great importance of this number in atomic physics, Dr. Born criticised the existing explanations of it.

Sir Arthur Eddington considers that it is associated with the number of degrees of freedom of a pair of electrons, and obtains the value 137. Dr. Born rejects this view, and seeks for an alternative explanation based on the new Born-Infeld-Pryce unitary field theory, which considers matter and field as one and the same. It involves a very large constant called 'the absolute field', which is the magnitude of the field in the centre of the electron. It is suggested that the number 137 is related to the neutralisation frequency of oscillation of a pair of electrons, one positive and the other negative (produced by light quanta passing the field of a nucleus), which approach and finally neutralise each other, emitting light quanta. Apparently the details of the calculation have not been worked out on Born's own theory, but by working on a somewhat similar theory Euler and Kockel (two pupils of Heisenberg) obtain the value $82 \cdot 4$. This differs considerably from the value $137 \cdot 2$, but Dr. Born considers the discrepancy not discouraging in view of the arbitrary assumptions made in the theory.

Dr. Born also uses the new field theory to explain the ratio of the proton and the electron, and obtains the number 2340 . The experimental value is $1846 \cdot 6$, and the theory of Sir Arthur Eddington gives the value $1847 \cdot 6$ (Proc. Roy. Soc., A, 134, 524 ; 1931). 